\title{
Frontières
}

\section{Deux textes et un témoignage sur le deuil et la résilience}

\section{Émilie C. Lévesque}

Volume 22, numéro 1-2, automne-printemps 2009-2010

Résilience et deuil

URI : https://id.erudit.org/iderudit/045038ar

DOI : https://doi.org/10.7202/045038ar

Aller au sommaire du numéro

Éditeur(s)

Université du Québec à Montréal

ISSN

1180-3479 (imprimé)

1916-0976 (numérique)

Découvrir la revue

Citer ce document

Lévesque, É. C. (2009). Deux textes et un témoignage sur le deuil et la résilience. Frontières, 22(1-2), 125-128. https://doi.org/10.7202/045038ar d'utilisation que vous pouvez consulter en ligne.

https://apropos.erudit.org/fr/usagers/politique-dutilisation/ 


\section{Deux textes et un témoignage sur le deuil et la résilience}

\author{
Émilie C. Lévesque, \\ auteure.
}

\section{SURMONTER SA PEUR ET VIVRE}

«La barre étale de la mer, blanche, à perte de vue, sur le ciel gris, la masse noire des arbres, en ligne parallèle derrière nous. » Seule couleur du tableau, ton maillot rouge ou plutôt, le sang qui rougit ton maillot. Le sang qui s'écoule hors de toi doucement, sans relâche. Pour une fois, tu iras au bout de ce que tu as commencé: l'anticoagulant que j'ajoute depuis des semaines dans le jus d'orange que je te presse chaque matin assurera la réussite de ton projet.

"Au loin une rumeur de fête, du côté du nouveau village.» La musique accompagne ton grand départ. Tu l'as si souvent demandé ce départ, si souvent tenté. Ton corps porte les marques que tes précédentes tentatives ont laissées. Chacune de ces blessures me brisait le cœur. Les rires et les feux d'artifice célèbrent aussi ton anniversaire. Tu as eu vingt-six ans aujourd'hui. Tu as essayé si souvent de quitter cette existence, je savais avoir trouvé le cadeau idéal pour toi. Même s'il m’était pénible.

«Leur fanfare se mêle au vent. » Je t'aime. J'ai eu le cœur brisé quand j'ai compris ce que je devrais t'offrir aujourd'hui. J'aurais voulu te garder, mais ç'aurait été égoïste. Mon amour n'aurait pu compenser ta crainte de rester vivante. Je vois la panique dans tes yeux quand tu comprends que tu iras jusqu'au bout. La panique qui t'a jusqu'ici empêchée de terminer ton adieu, ta libération. Panique à cause de la peur de l'inconnu, mais tu recommençais toujours, déçue de n'avoir pas réalisé ce que tu avais tenté. Cette fois, par amour pour toi, je t'ai aidée à y arriver. À surmonter ta peur. J'ai tout fait pour t'empêcher de rater ton suicide.

Pardonne-moi si je n'ai pas compris que cette fois, tu désirais vivre ${ }^{1}$.

\section{LE DEGRÉ ZÉRO DE L'EXISTENCE}

Au commencement était le vide et l'esprit de la femme était porté par l'absence.

On ne peut pas être et avoir été. Parfois, il faut détruire ce qu'on a été avant de commencer enfin à être. Il faut oublier ce qui était, cesser de rabâcher le passé pour se concentrer sur ce qui viendra. L'histoire peut servir de guide et de rappel, mais ne doit pas remplacer le présent.

Le degré zéro de l'existence, c'est remettre le compteur au début, effacer ce qui précédait, fuir la réalité douloureuse dans une presque mort dont on ne veut pas vraiment parce qu'elle signifierait la fin de tout.

Le degré zéro, c'est être suspendue au-dessus de soi et se demander qui on est, ce qu'on fait là. C'est réapprendre l'existence.

Elle les entend parler autour d'elle. Pour le moment, pendant leurs palabres sur son état, eux qui prétendent savoir, mais ignorent tout, elle a la sensation de baigner dans la crème. Pendant qu'ils s'énervent et s'inquiètent, elle se repose dans un calme moelleux. Son corps semble glisser au milieu d'une épaisse crème chaude qui l'enrobe, la protège, la caresse.

Elle s'étire et plonge dans cet univers de rêve. Les sons s'alourdissent, les discours deviennent confus et son corps se libère de sa gangue. Elle devient éthérée, immatérielle. Elle cesse d'avoir été.

Sensation physique d'un corps terrestre isolé de tous stimuli. Comme suspendu entre ciel et terre, sous une voilure, dans l'absolu du silence. Entre deux airs, entre deux eaux, entre deux réalités. Immobilité. Inertie. Fixité. Symphonie en fugue majeure. Non-être. Néant.

Être dans un rêve et se regarder ne plus évoluer. On ne vit jamais le rêve: on y assiste, tout simplement. Le rêve est une mise en scène, un saut en parachute. La matière s'efface pour laisser place au spectacle. Mais de spectacle ici, il n'y en a pas. Que la matière effacée. 
Son corps attend, allongé sur un lit qui n'est pas le sien, un lit petit, dur et inconfortable; mais c'est sans importance parce qu'elle ne ressent rien. Des appareils dominent le lit. Une aiguille reliée à un soluté est plantée dans le gras du pouce de sa main droite, au niveau de l'articulation carpo-métacarpienne.

On alimente le corps de la femme, corps qu'elle n'habite plus.

Est-ce cela la mort? Mais pourquoi nourrirait-on la mort?

Hier encore elle était présente, elle vivait, elle existait. Hier, ou peut-être la semaine dernière ou le mois passé. Avant. Avant qu'elle ne mette le compteur dans un vacuum, qu'elle n'élimine les références. Mourir, mais sans mourir. Faire semblant de, jouer à, aller au bord, aux frontières, mais sans regarder de l'autre côté. Disparaître au présent pour revenir autrement. Rompre le contact avec la réalité si douloureuse. Rejeter sa vie en préservant son être. Couper le contact, rompre l'alimentation.

"Calmer enfin dans le sommeil les affreux battements du cœur / Quelle conclusion des maux héréditaires seraient plus dignement souhaités ? ${ }^{\text {» }}$

La nuit s'est installée, c'est sombre et paisible. L'univers dans son ensemble est amorti, tandis qu'elle plane toujours au-dessus de tout. Jusqu'à ce moment où elle a à nouveau l'impression de s'estomper, de se brouiller. De s'effacer.

$$
* * *
$$

Au commencement était le vide. Le corps reposait sur le lit et l'esprit de la femme était porté par les eaux. Les ténèbres l'envahirent et la femme y sombra. L'esprit et la matière ne firent plus qu'un.

Une quête de mort qui se voulait une fin, mais surtout une renaissance. La voilà de retour à la matrice, baignant dans un liquide amniotique virtuel, suspendue entre deux. Corps porté à la dérive en quête d'une remise au monde.

Ses sens se réveillent. Odeur de javel, de maladie et de médicaments. Atmosphère terne. Quelques sons lui parviennent étouffés: des semelles de crêpe sur le linoléum du plancher, le tintement de l'ascenseur, une télévision ou un poste de radio qui diffuse un truc lambda avec des dialogues insipides. Des discussions qu'elle n'arrive pas à suivre. Une infirmière qui passe en poussant un plateau à roulettes. Puis le bruit de pas un peu plus prononcés. Des pas qui se rapprochent.

Elle referme les yeux et cesse de bouger. Elle n'est pas prête à affronter le regard de quelqu'un d'autre, son anxiété, ses questions. Ou ses réponses. Elle veut d'abord trouver ses repères, emplir le bocal de ses souvenirs. Comprendre ce qui lui est arrivé et surtout, pourquoi.

Immobilité, toujours. Elle adopte volontairement l'inertie globale, le statu quo. Elle ne bouge pas, respire à peine. Quelqu'un entre dans sa chambre, se rapproche du lit. Les pas cessent. Une présence immobile qui la regarde. Ses yeux sont fermés, mais les autres sens s'en donnent à cœur joie. Elle a l'impression que les terminaisons nerveuses de son épiderme multiplient par cent les sensations de sa peau. Elle sent la chaleur du regard. Elle perçoit le mouvement de l'air qui lui indique que la personne se penche vers elle. L'odeur de l'homme chatouille ses narines et elle sait qui est là. Quand il parle, son souffle réchauffe sa joue.

- Je t'aime. Je dois partir, mais je reviendrai demain.

Des lèvres embrassent la peau que son haleine vient de réchauffer. Puis, le bruit des pas reprend et elle imagine son déplacement dans le corridor. Elle attend d'être certaine qu'il soit parti avant de rouvrir les yeux.

$$
* * *
$$

Elle est. Libérée de ce qu'elle a été, elle regagne son corps, réintègre son esprit, elle s'habite à nouveau. Reprend vie. Cette vie à laquelle elle ne peut se rattacher, qui lui semble soudain se dissoudre entre ses doigts. Elle a peur.
Peur de se dire adieu. Peur de se quitter avant même de s'être retrouvée, peur de se sentir mourir sans pouvoir rien y changer. Le visionnement s'amorce, le film d'une vie avec elle dans le rôle principal. Un film muet en noir et blanc avec le bruit du rouage des projecteurs en arrière-plan.

Angoisse. Et si tout ça n'était que le jeu d'une déité cruelle décidée à lui faire regretter son geste en lui faisant voir tout ce qu'elle a perdu? Et si après avoir vu sa vie défiler devant ses yeux, il y avait cette lumière au loin, lueur irrésistible qui l'entraînerait loin dans la mort?

Les ténèbres la recouvrent et elle cesse d'être.

\section{TÉMOIGNAGE - LA VIE EST CRUELLE, ET PUIS APRÈS?}

C'est dur la vie. Elle est injuste, elle est difficile, elle bloque les chemins, elle nous renverse. À nous de nous relever, de passer par-dessus. On peut choisir aussi de rester là, effondré, désarmé. On peut choisir de rendre les choses plus difficiles encore.

Ma vie a pris fin il y a dix-neuf ans. Ma deuxième vie a débuté à ce moment. J'aurais pu mourir quand c'est arrivé. J'aurais pu crever dans les années qui ont suivi. J'ai survécu, j'ai surmonté, j'ai fait des deuils et je continue à en faire.

Parfois, je me demande ce que je fais là. Je songe à ce que j'ai perdu, à ce que j'ai raté, à ce que je n'arrive pas à faire. Et je me sens déprimée.

Mais toujours, il y a cette rage de vivre. Entêtement primitif. Instinct de survie. Fierté égocentrique. C'est vrai, j'ai eu de la chance. J'en ai bavé, aussi. Beaucoup, parfois. Mais je m'en suis bien tirée.

J'étais sur la fin de ma quinzième année. Un vélo, une voiture, une rencontre fracassante. Soins intensifs, trépanation d'urgence, coma. La descente aux enfers le long de l'échelle de Glasgow. Chances de survie: inconnues. État prévisible au réveil : inconnu. Un pouce cassé. Un traumatisme craniocérébral sévère. Des machines partout pour me garder en vie.

Trois semaines plus tard, dans une chambre à l'étage. Pas encore tout à fait consciente, mais les fonctions minimales sont assurées: je respire seule. Je ne parle pas. Je ne marche pas. Je ne mange pas seule. Je ne sais rien faire. Il faut tout reprendre.

Aujourd'hui, j'ai l'air d'une personne normale. Mais je demeure une personne handicapée et je le serai toute ma vie. Un traumatisme craniocérébral comporte une variété infinie de variables. Des comportements erratiques. Des troubles neurologiques. Une absence d'inhibition. Et d'autres encore. Déclinés de toutes les manières possibles selon les victimes. Sauf que ça ne paraît pas toujours.

Handicap invisible.

J'ai eu de la chance. Ma récupération a été extrêmement rapide. J'ai passé moins de deux mois à l'hôpital, j'ai fait de la réadaptation en externe pendant trois mois à raison de trois jours par semaine, puis je suis passée à une journée aux trois semaines pendant six mois.

Quatre mois et demi après mon accident, je retournais sur les bancs de l'école pour terminer mes études secondaires. Évidemment, j’avais un programme allégé: je ne suivais que quatre cours, j'en ai échoué un et j'en ai passé un autre de justesse. Mais j'ai obtenu mon D.E.S.

J'ai lu sur les traumatismes crâniens pendant cette année là. J'ai compris ce que je lisais, mais entre comprendre ce qui était écrit et ce que ça impliquait pour moi, il y avait une marge.

Je suis ensuite partie étudier à l'extérieur. Ce fut peut-être une erreur, mais peut-être aussi que j'avais besoin de ça pour m'en sortir seule. Qui peut prétendre anticiper sur les choix et décisions des autres? Je m'en suis sortie parce que je possède en moi les ressources nécessaires, mais aussi parce qu'on m'a poussée. On m'a obligée à me lever et à marcher, à refaire des 
ronds avec un crayon, à enfiler du fil dans le chas d'une aiguille, à poursuivre mes études. On m'a aidée à avancer.

Vous savez, un des éléments les plus difficiles à gérer après un traumatisme craniocérébral, c'est qu'on a changé, mais on ne s'en rend pas compte. On se croit normal, comme avant. Mais les gens qui nous connaissaient voient bien qu'on a changé. Et ils n'aiment pas ça. On croit agir normalement, comme les autres, mais ce n'est pas le cas. On aimerait être accepté; on est jugé.

Alors vient le rejet. L'abandon. L'incapacité à créer ou entretenir des relations interpersonnelles. Quand on a seize ou dix-sept ans, c'est extrêmement difficile à vivre. Et il y a l'alcool aussi. La compulsion. Le déni (non, moi je suis correcte, je suis guérie, je n'ai aucun problème, tout va bien madame la marquise). Et l'anosognosie, terme de neuropsychologie qui implique une méconnaissance par le malade de son état. Alors on fait des erreurs, encore et encore. On se blesse, mais on ne comprend pas et on se blesse encore. On recommence, encore et toujours.

Après un an à l'extérieur, je suis revenue, j'ai complété mon DEC dans un autre cégep avant d'aller à l'université. J'ai mis cinq ans à faire mon BAC, mais je l'ai fait. À l'occasion, je travaillais, mais ma relation avec le monde du travail était extrêmement difficile, en raison de mes atteintes, même si je ne le savais pas encore.

J'ai eu mon accident à la fin de ma quinzième année. Il y a eu ensuite huit ans d'études, de quêtes, d'échecs, de difficultés, de réussites, de rejets, de souffrance et de vie. Je les appelais mes huit ans d'enfer. Un ami m'a suggéré le terme de huit ans d'adaptation. Je l'en remercie, il m'a aidé à faire la paix avec cette période.

Puis j'ai rencontré mon partenaire de vie. Un homme qui m'a rencontrée et acceptée comme j'étais, sans condition. Qui a reçu mon amour et m'a aimée en retour. Nous sommes mariés depuis dix ans et nous avons trois filles magnifiques.

\section{INNÉE OU ACQUISE ?}

Qu'est-ce qui fait qu'on est résilient? Vous connaissez probablement des gens qui, à la moindre épreuve, sont dépassés, qui perdent leurs moyens dès que les choses ne vont plus comme ils le veulent. Vous connaissez aussi des gens qui retombent sur leurs pattes et effectuent des virages à 90 degrés sans broncher. Des gens qui, après une enfance difficile, sombreront dans la délinquance et le crime et d'autres qui réussiront dans la vie. Est-ce à dire que la résilience, on l'a ou on ne l'a pas?
Peut-être. Mais je crois qu'on peut la favoriser. À l'ami déprimé, on peut faire voir le bon côté des choses. On apprend à nos enfants à ne pas se laisser freiner par les erreurs et les échecs. On leur apprend l'importance de la persévérance.

La résilience, c'est parfois inné, mais c'est parfois acquis. Et pour l'acquérir, il faut être guidé.

Si mes parents, ma famille, les intervenants, infirmières, médecins et les autres ne m'avaient pas poussée, je serais probablement restée au même niveau pendant longtemps, sans progresser. On m'a donné le coup de pouce dont j'avais besoin pour retrouver le goût de me battre, de me dépasser, de continuer à avancer dans la vie. À la base et pour continuer, il y avait ce que j'étais, profondément. Mais on m'a peut-être aidée à le trouver.

Les gens se disent impressionnés par mon énergie, ma bonne humeur, ma persévérance. Je leur réponds que je n'ai simplement pas de temps à perdre à me mettre en colère contre moi, à rabâcher mes échecs et mes limites: je ne ferais que ça. Il m'est arrivé si souvent de me tromper, d'échouer, de manquer mon coup, de faire des erreurs, que si je m'arrêtais à chaque fois, je cesserais d'avancer.

On en revient toujours à l'histoire du verre d'eau : estil à moitié plein ou à moitié vide?

Évidemment, tout ça ne s'est pas fait en une journée, ni en un mois, ni même en une année. Parce que recommencer à marcher, à parler, à écrire, ce n'est pas de la résilience, c'est de la réadaptation. La réadaptation est un premier pas vers la résilience, mais ce n'est pas la même chose.

Après (pendant) la réadaptation vient le refus (je parle de mon expérience). Il y a eu l'accident, il y a eu l'hôpital, maintenant on veut reprendre notre vie comme si rien ne s'était passé. Et ça non plus ce n'est pas de la résilience, c'est du déni.

La résilience commence avec la reconnaissance de ce qui est arrivé, puis son acceptation. C'est comprendre qu'il y a une vie après, une vie différente, une vie marquée par des stigmates, mais une vie quand même.

Parce qu'il y a eu le traumatisme craniocérébral, mais il y a eu surtout toute une série de deuils qui ont suivi, qui ont été plus ou moins bien vécus : deuil de la personne qu'on était avant, deuil des amis qu'on avait, deuil des relations diverses, qui se brisent et parfois revivent différemment, échecs répétés dans différents domaines, expériences difficiles dans l'apprentissage de la nouvelle vie/identité... 
J'ai eu mon accident à l'âge de quinze ans. Les huit années qui ont suivi ont été, malgré mes accomplissements (j'ai obtenu mon bac au terme de ces huit ans, entre autres), très difficiles. Après, une autre phase de ma vie a commencé avec la rencontre de mon amoureux et la naissance de mes enfants. La stabilité émotionnelle que m'apportaient l'amour de mon mari et la naissance de mes enfants ont été des atouts importants pour ma résilience.

Dans les années qui ont suivi, j'ai fait une demande de réévaluation auprès de la SAAQ, pour faire reconnaître mes limitations et affects (mon dossier avait par erreur été classé trauma léger) et j'ai aussi obtenu un suivi neuropsychologique qui a duré cinq ans et demi. Le fait de voir reconnaître mes limitations leur donnait une légitimité: je suis passée d'une personne nulle et incapable, dans ma tête, à une personne ayant des limitations réelles. Mon estime personnelle, rudement malmenée, en a profité. Ma neuropsychologue, de son côté, m'a aidée à comprendre mon traumatisme craniocérébral - syndrome frontal (appellation signifiant que le gros du choc a été absorbé par le front et que les zones les plus atteintes de mon cerveau sont situées à cet endroit apportant telle réaction, tel comportement, telle limitation).

Pouvoir enfin comprendre m'a énormément aidée.

Comprendre d'où viennent certains comportements aide à les apprivoiser. À penser et faire autrement.
Et c'est là que la résilience embarque. Il faut d'abord reconnaître ses deuils. Reconnaître, accepter et laisser aller. Ensuite, on peut s'asseoir et choisir de s'apitoyer sur son sort et de jouer à la victime ou bien retrousser ses manches et poursuivre sa vie. La résilience, c'est dire oui j'ai souffert, oui j'ai été blessée, oui j'ai été victime, mais maintenant je m'en suis sortie et le reste de ma vie m'appartient.

Aujourd'hui, j'ai trente-quatre ans. J'ai publié un premier roman en 2007 et je continue à écrire (j'ai aussi publié deux nouvelles et mon deuxième roman est en lecture chez un éditeur). J'ai également décidé de réaliser mon rêve et d'apprendre à jouer du piano. Je souffre régulièrement de maux de tête (qui ont diminué d'intensité depuis que je prends un médicament contre l'anxiété), je dois constamment gérer mon énergie (je dispose d'une quantité limitée pour la journée, alors je dois l'utiliser avec parcimonie), j'ai appris et j'apprends encore à connaître mes limites. Mais surtout, je vis. Et j'aime.

\section{Notes}

1. Les citations sont tirées d'un roman d'Anne Hébert. Chaque phrase est la première des trois premiers paragraphes du livre Les fous de Bassan.

2. Shakespeare, Hamlet, traduction libre. 